\title{
Perfil epidemiológico do câncer do colo do útero no Estado do Piauí
}

\author{
Epidemiological profile of cervical cancer in the State of Piauí \\ Perfil epidemiológico del cáncer de cuello uterino en el Estado de Piauí
}

Recebido: 26/09/2021 | Revisado: 02/10/2021 | Aceito: 06/10/2021 | Publicado: 10/10/2021

Walkiria Brenda de Sousa Bezerra
ORCID: https://orcid.org/0000-0003-2560-7557
Universidade Federal do Piauí, Brasil
E-mail: walbrenda@ @otmail.com
Paulo Pedro do Nascimento
ORCID: https://orcid.org/0000-0003-2626-7582
Universidade Federal do Piauí, Brasil
E-mail: ppfarmaco@globo.com
Stephany Suelen de Castro Sampaio
ORCID: https://orcid.org/0000-0003-0884-8412
Universidade Federal do Piauí, Brasil
E-mail: stephany_suelen@ @otmail.com

\begin{abstract}
Resumo
O presente estudo tem o objetivo de conhecer o perfil epidemiológico do câncer do colo do útero no Estado do Piaú para possibilitar a compreensão de alguns aspectos relacionados a essa patologia que possui alto potencial preventivo. Trata-se de um estudo com apreciação documental, epidemiológico, retrospectivo, descritivo, com abordagem quantitativa dos dados, obtidos no Sistema de Informação do Câncer (SISCAN), referente ao ano de 2019. Foram analisadas as seguintes variáveis: total de exames, faixa etária, escolaridade, período preventivo, motivação para o exame e realização da citologia anterior. O levantamento de informações mostrou que 77.841 exames foram realizados, a maior quantidade concentrou-se na faixa etária de 35 a 39 anos e 99,58\% (77.514) tiveram como motivação o rastreamento. Neste estudo, $73,98 \%$ (57.586) das mulheres afirmam terem realizado um exame preventivo anteriormente, $15,81 \%$ (12.306) não realizaram o teste, $8,29 \%$ (6.453) não sabem e 1,92\% (1.494) não apresentavam informação na ficha. Relacionado a escolaridade, o SISCAN não forneceu dados satisfatórios para análise. O rastreamento do CCU é uma ação complexa, o número de mulheres acometidas por essa patologia é considerado alto e significativo. É notória a necessidade de conhecimento sobre o tema em questão para o fortalecimento e redirecionamento das políticas públicas de controle do CCU.
\end{abstract}

Palavras-chave: Neoplasias do colo do útero; Teste de Papanicolau; Epidemiologia; Sistema único de saúde.

\begin{abstract}
This study aims to understand the epidemiological profile of cervical cancer in the State of Piauí to enable the understanding of some aspects related to this pathology, which has a high preventive potential. This is a study with documentary, epidemiological, retrospective, descriptive analysis, with a quantitative approach to data obtained from the Cancer Information System (SISCAN), for the year 2019. The following variables were analyzed: total number of exams, range age, education, preventive period, motivation for the examination and performance of previous cytology. The survey of information showed that 77,841 exams were carried out, the largest amount was concentrated in the age group of 35 to 39 years old and $99.58 \%(77,514)$ were motivated by tracking. In this study, $73.98 \%$ $(57,586)$ of the women claim to have had a preventive exam before, $15.81 \%(12,306)$ did not undergo the test, $8.29 \%$ $(6,453)$ do not know and $1.92 \%(1,494)$ do not presented information in the form. Regarding education, SISCAN did not provide satisfactory data for analysis. CC screening is a complex action, the number of women affected by this pathology is considered high and significant. The need for knowledge on the subject in question to strengthen and redirect public policies for the control of the $\mathrm{CCU}$ is notorious.
\end{abstract}

Keywords: Cervical neoplasms; Papanicolaou test; Epidemiology; Unified health system.

\section{Resumen}

Este estudio tiene como objetivo conocer el perfil epidemiológico del cáncer de cuello uterino en el estado de Piauí para permitir comprender algunos aspectos relacionados con esta patología, que tiene un alto potencial preventivo. Se trata de un estudio con análisis documental, epidemiológico, retrospectivo, descriptivo, con enfoque cuantitativo de los datos, obtenidos del Sistema de Información del Cáncer (SISCAN), para el año 2019. Se analizaron las siguientes variables: número total de exámenes, rango de edad, educación, período preventivo, motivación para el examen y realización previa de citología. La encuesta de información arrojó que se realizaron 77,841 exámenes, la mayor cantidad se concentró en el grupo de edad de 35 a 39 años y el $99.58 \%(77,514)$ fueron motivados por el rastreo. En este estudio, el 73,98\% $(57,586)$ de las mujeres afirman haberse sometido a un examen preventivo antes, el 15,81\% 
$(12,306)$ no se sometió a la prueba, el 8,29\% $(6,453)$ no sabe y el 1,92\% $(1,494)$ no presentó información. en la forma. Con respecto a la educación, el SISCAN no proporcionó datos satisfactorios para el análisis. El cribado de CC es una acción compleja, el número de mujeres afectadas por esta patología se considera alto y significativo. Es notoria la necesidad de conocimiento sobre el tema en cuestión para fortalecer y reorientar las políticas públicas para el control de la UCC.

Palabras clave: Neoplasias cervicales; Prueba de Papanicolau; Epidemiología; Sistema único de salud.

\section{Introdução}

O câncer do colo do útero (CCU), também denominado de câncer cervical, é um dos tipos mais frequentes e uma importante questão de saúde pública, representando cerca de 7,4\% da quantidade de novos casos de câncer que acometem as mulheres no Brasil e a quarta causa de mortalidade nesse grupo. Segundo o Instituto Nacional de Câncer, o INCA (2020), para o estado do Piauí, estima-se 19,82 novos casos de CCU para cada 100 mil mulheres (Brasil, 2013).

De acordo com o INCA (2020), o CCU caracteriza-se por um crescimento desordenado das células que revestem o útero, envolvendo o tecido subjacente (estroma), podendo invadir estruturas que estejam próximas ou distantes da área afetada. Existem dois tipos principais de carcinomas invasores do colo do útero: o carcinoma epidermoide e o adenocarcinoma. O carcinoma epidermoide atinge o epitélio escamoso e é o tipo mais incidente, representando um total de $90 \%$ dos casos diagnosticados. O adenocarcinoma, é um tipo mais raro que acomete o epitélio glandular e representa um total de $10 \%$ dos casos (Brasil, 2013).

O Papilomavírus Humano (HPV) considerado sexualmente transmissível, representa um grupo de vírus muito comum no mundo, sendo o CCU a doença mais frequente relacionada a esse vírus (OMS, 2019). O HPV é citado pela literatura como condição primordial ao crescimento de lesão intraepitelial de alto grau e câncer invasivo do colo do útero, tendo em vista que o DNA viral do HPV está presente em aproximadamente 90\% das lesões pré-neoplásicas (Pinto, Fuzii \& Quaresma, 2011). Dessa forma, acredita-se que o câncer é provocado por infecção constante via subtipos oncogênicos do HPV (Lopes \& Ribeiro, 2019).

A infecção pelo HPV é uma condição necessária para o desenvolvimento de CCU, no entanto não representa uma causa suficiente para o desenvolvimento desse tipo de câncer (Thuler, Bergmann \& Casado, 2012). Outros Fatores colaboram para a evolução do CCU, dentre os mais conhecidos estão: idade; desnutrição; situação socioeconômica; início precoce da atividade sexual e múltiplos parceiros; histórico de outras doenças sexualmente transmissíveis; hábitos inadequados de higiene; tabagismo e o uso prolongado de pílulas anticoncepcionais (De Carvalho, et al., 2018); (INCA, 2020).

O diagnóstico precoce é a forma mais eficaz de controle do $\mathrm{CCU}$, através do tratamento das lesões precursoras e tumorais invasoras em seus estágios iniciais que é quando o potencial de cura é expressivo (Rangel, Lima \& Vargas, 2015). Apesar da alta incidência da doença, essa neoplasia possui um desenvolvimento considerado lento e as alterações celulares que podem desencadeá-la são facilmente detectadas no exame preventivo (Silva, 2019). Nesse contexto, o CCU possui um método efetivo para o seu rastreamento, o exame de Papanicolau, conhecido também como citologia oncótica, colpocitologia oncótica e citopatológico, que aumenta as possibilidades terapêuticas à proporção que colabora para o diagnóstico prévio das lesões (Kose \& Naki, 2014); (Rangel, Lima \& Vargas, 2015).

Nesse sentido, o Ministério da Saúde (MS) desenvolveu o Sistema de Informação do Câncer (SISCAN). Este sistema está associado ao Cadastro Nacional de Cartão de Saúde que permite a identificação da mulher com diagnóstico confirmado. As informações inseridas no SISCAN encontram-se disponíveis em tempo real pela Internet, viabilizando às Unidades de Saúde da Família (USFs) e Unidades Básicas de Saúde (UBSs) a realizarem exames de rastreamento, segundo periodicidade e faixas etárias indicadas (Brasil, 2017).

Diante do exposto, o presente estudo teve como objetivo conhecer o perfil epidemiológico do CCU no Estado do Piauí a partir da análise das citologias que foram realizadas no ano de 2019 e registradas no SISCAN e assim possibilitar a 
compreensão de alguns aspectos relacionados a essa patologia que possui alto potencial preventivo, mas ainda acomete as mulheres com números cada vez mais expressivos.

\section{Metodologia}

Trata-se de um estudo retrospectivo, descritivo, epidemiológico e com abordagem quantitativa, com dados coletados referentes ao Estado do Piauí no ano de 2019. Desta forma, este estudo epidemiológico caracteriza-se por ser, sobre o perfil do câncer do colo do útero no Estado do Piauí, através de pesquisa documental (Pereira, et al., 2018)

Os dados foram coletados no SISCAN- Sistema de Informação do Câncer (colo do útero e mama), que é uma versão em plataforma Web do Departamento de Informática do Sistema Único de Saúde (DATASUS), que integra os sistemas de informação do Programa Nacional de Controle do Câncer do Colo do Útero (SISCOLO) e o Programa Nacional de Controle do Câncer de Mama (SISMAMA). Essa base de dados apresenta o registro de todos os exames citopatológicos do colo do útero realizados pelo Sistema Único de Saúde (SUS). Para a realização do estudo foram considerados todos os dados de exames citopatológicos do colo do útero realizados no ano de 2019, em mulheres residentes no Estado do Piauí.

A organização dos dados para análise foi feita através do programa Microsoft Excel ${ }^{\circledR} 2016$ para processamento das informações, sendo discutidas à base do referencial bibliográfico, a partir das bases de dados: Google Acadêmico, Medline, Pubmed, Lilacs e SciElo. Os dados foram analisados quantitativamente e de maneira descritiva.

A análise dos dados foi realizada considerando-se as seguintes variáveis: total de exames realizados no Estado do Piauí, faixa etária, escolaridade, período do preventivo, motivo da realização do exame e realização de citologia anterior. Para melhor visualização e interpretação dos resultados, os dados foram agrupados em gráficos e tabelas confeccionados a partir dos programas Microsoft Excel $^{\circledR} 2016$.

Por tratar-se de análise de dados secundários de domínio público, não houve necessidade de apreciação em Comitê de Ética em Pesquisa (CEP), pois este estudo não promove intervenção nas pessoas do estudo, estando de acordo com a resolução de número 466 de 12 de dezembro de 2012 do Conselho Nacional de Saúde (CNS), que trata das Diretrizes e Normas Regulamentadoras de Pesquisas Envolvendo Seres Humanos.

\section{Resultados}

O levantamento de informações a partir da base de dados do DATASUS mostrou que 77.841 exames citopatológicos foram realizados no Piauí no ano de 2019, com uma média de 6.487 exames por mês, o que evidenciou um aumento de 20,97\% na taxa de realização dos exames e assim uma maior cobertura e aumento do rastreamento quando comparado ao ano anterior (2018), no qual foram realizadas 64.347 citologias oncóticas.

De acordo com a Figura 1, a maior quantidade de exames realizados no Estado do Piauí concentrou-se na faixa etária de 35 aos 39 anos (10.121 exames), havendo um considerável declínio de realização de exames por mulheres concentradas na faixa etária acima de 65 anos. Ressalta-se ainda que a incidência desse tipo de câncer no Brasil é evidenciada entre mulheres de 20 a 29 anos, estando o maior risco na faixa etária de 45 a 49 anos (Rico \& Iriart, 2013). 
Figura 1- Distribuição do total de exames citopatológicos realizados no Piauí de acordo com a faixa etária $(\mathrm{n}=77.841)$.

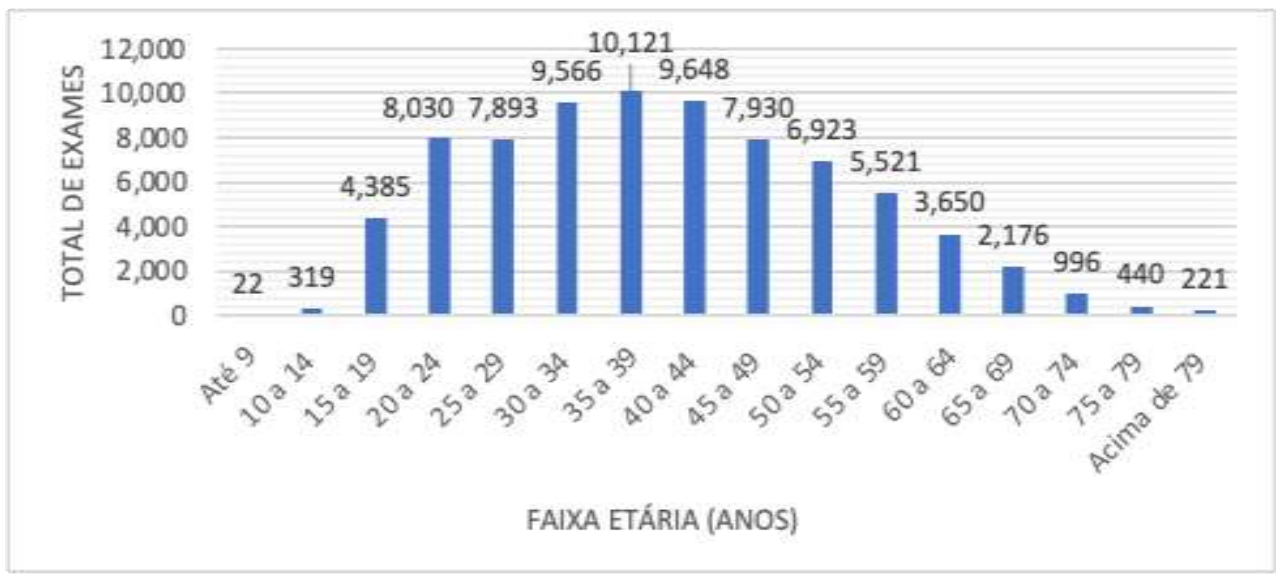

Fonte: DATASUS (2020).

A Figura 1 ainda mostra que foram realizados 319 exames em mulheres na faixa etária de 10 aos 14 anos, que compreende o período de início da adolescência que é de 12 aos 18 anos de idade. É possível observar também um aumento da realização do exame nas faixas etárias seguintes.

O período do preventivo é o tempo calculado, pelo sistema, entre o ano de realização do último exame citopatológico do colo do útero, informado pela paciente, e a data de realização do exame atual (Brasil, 2017). A Figura 2 mostram que foram realizados 4.916 testes no mesmo ano; 30.834 exames após um ano da realização do primeiro teste; 12.263 após dois anos; 4.486 após três anos e 2.125 após quatro anos ou mais.

Figura 1- Distribuição do total de exames citopatológicos realizados no Piauí de acordo com o período do preventivo $(n=54.624)$.

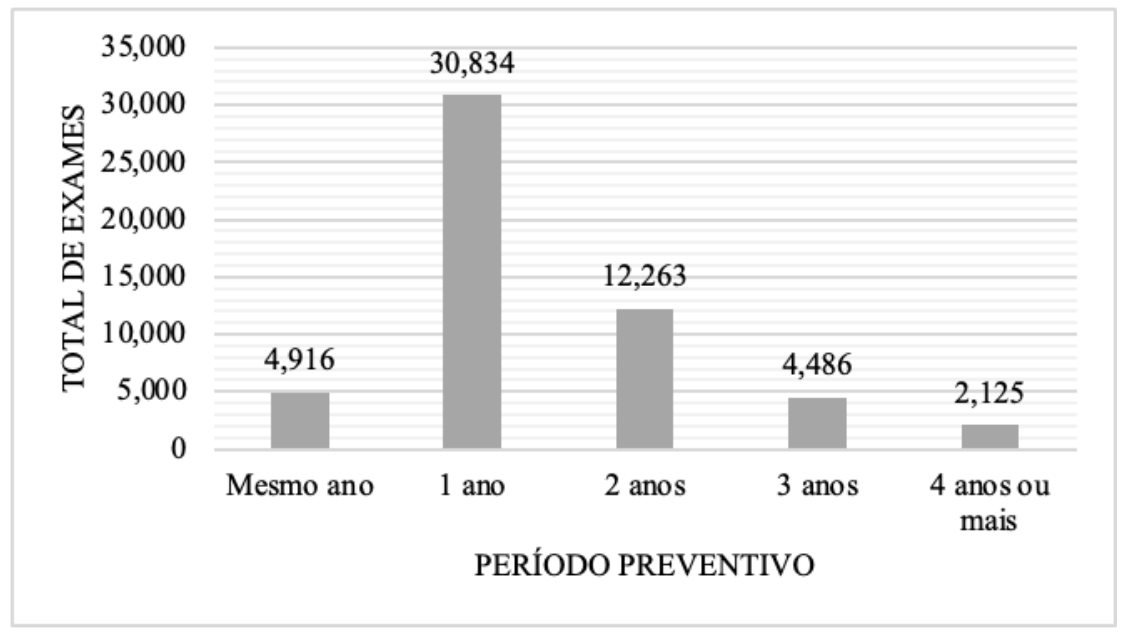

Fonte: DATASUS (2020).

Segundo o Ministério da Saúde (2017), citologia anterior corresponde à informação fornecida pela mulher e preenchida no formulário de haver ou não realizado anteriormente um exame citopatológico do colo do útero. Neste estudo, de acordo com a Tabela 1, 73,98\% das mulheres afirmam terem realizado um exame preventivo anteriormente; $15,81 \%$ não realizaram o teste; $8,29 \%$ não sabem e 1,92\% não apresentavam informação na ficha. Ao correlacionarmos esse aspecto a faixa etária observa-se que maior parte de mulheres que afirmam não terem realizado um exame anteriormente compreendem o 
intervalo de 15 a 29 anos, ou seja, quanto menor a idade maior foi o risco de não ter realizado o exame preventivo do CCU (Brasil, 2017).

Tabela 1- Realização de citologia anterior de acordo com a faixa etária no Piauí- 2019 (n=77.841).

\begin{tabular}{c|cccc}
\hline \multicolumn{5}{c}{ Citologia Anterior } \\
\hline $\begin{array}{c}\text { Faixa Etária } \\
\text { (anos) }\end{array}$ & Sim & Não & Não Sabe & Sem informação na ficha \\
\hline Até 9 & 7 & 12 & 2 & 1 \\
10 a 14 & 48 & 234 & 29 & 8 \\
15 a 19 & 1.691 & 2.375 & 265 & 54 \\
20 a 24 & 4.923 & 2.379 & 603 & 125 \\
25 a 29 & 5.817 & 1.319 & 627 & 130 \\
30 a 34 & 7.577 & 1.126 & 703 & 160 \\
35 a 39 & 8.172 & 1.029 & 742 & 199 \\
40 a 44 & 7.694 & 945 & 810 & 175 \\
45 a 49 & 6.297 & 766 & 692 & 87 \\
50 a 54 & 5.532 & 659 & 580 & 97 \\
55 a 59 & 4.347 & 540 & 547 & 62 \\
60 a 64 & 2.825 & 373 & 355 & 35 \\
65 a 69 & 1.581 & 260 & 273 & 17 \\
70 a 74 & 688 & 158 & 115 & 14 \\
\hline 75 a 79 & 276 & 79 & 68 & $\mathbf{1 . 4 9 4}$ \\
\hline Acima de 79 & 113 & 50 & 44 & \\
\hline Total & $\mathbf{5 7 . 5 8 8}$ & $\mathbf{1 2 . 3 0 4}$ & $\mathbf{6 . 4 5 5}$ & \\
\hline
\end{tabular}

Fonte: DATASUS (2020).

Para o Sistema de Informação do Câncer (2017), os motivos para a realização do exame estão divididos em três categorias: rastreamento, repetição (exame alterado ASC-US/Baixo Grau) e seguimento para acompanhamento ou após tratamento de uma lesão. Conforme a Figura 3, 99,58\% dos exames realizados tiveram como motivação o rastreamento; 0,23\% dos exames foram realizados com indicação de repetição, devido à presença de alterações consideradas de baixo grau e 0,19\% dos exames foram realizados para fins de seguimento.

Figura 2- Motivo de realização do exame distribuído pelo total de exames citopatológicos realizados no Piauí $(n=77.841)$.

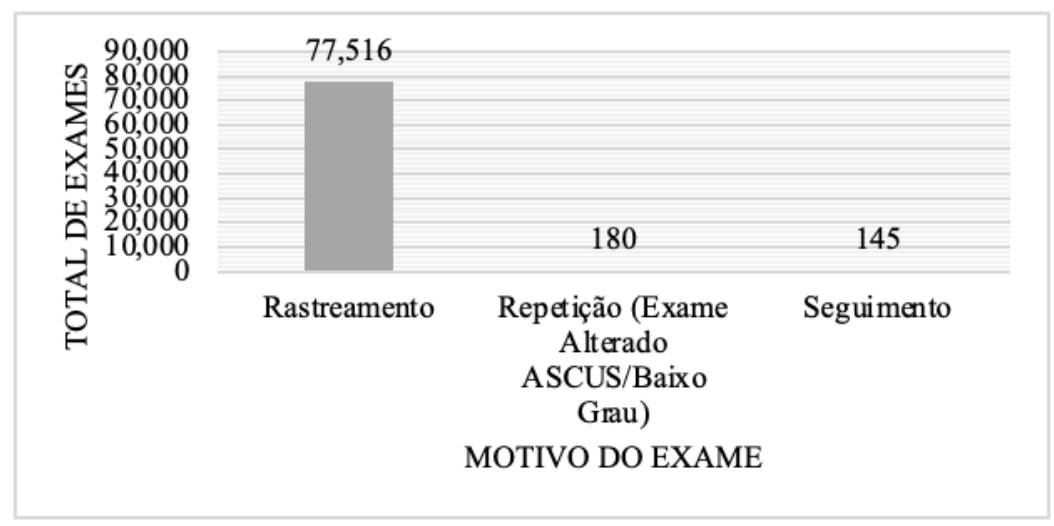

Fonte: DATASUS (2020). 
A frequência dos laudos de ASC-US é considerada um indicador da qualidade dos exames de um serviço. Com as normatizações recentes nos critérios diagnósticos, os resultados de ASC-US devem estar entre 3\% e 5\% do total de esfregaços avaliados. Sendo assim, por representarem 0,23\% dos exames, os laudos encontram-se dentro das especificações. A ocorrência de percentuais maiores sugere excesso de diagnósticos das alterações reativas, benignas, inflamatórias e reparativas (Backes, et al., 2014).

O SISCAN não fornece dados satisfatórios a respeito da escolaridade das mulheres que realizaram o exame de Papanicolau no Estado do Piauí no referido ano de execução do estudo, o que prejudica o entendimento acerca desse aspecto que é determinante para o desenvolvimento de medidas e ações que minimizem os fatores de risco e o progresso do CCU.

\section{Discussão}

O CCU é uma patologia com elevado índice de prevenção, tendo como método efetivo para o seu rastreamento o exame de Papanicolau que é de extrema importância para a saúde da mulher. Contudo, a realização deste exame de prevenção permite reduzir em até $70 \%$ a mortalidade por CCU na população de risco, pois esta neoplasia tem um desenvolvimento lento, e as alterações celulares que podem desencadeá-las são facilmente descobertas no exame preventivo (Araújo, Luz \& Ribeiro, 2011); (Nobrega, et al., 2014).

De acordo com a OMS (2012), uma abrangência de $80 \%$ da população de risco pelo exame preventivo seria suficiente para restringir de maneira significativa a ocorrência e a mortalidade pelo câncer cervical. O exame de Papanicolau tem sido mundialmente reconhecido como método seguro e eficaz para a identificação precoce desse tipo de câncer. A efetividade da detecção prévia de lesões precursoras por meio desse exame, concomitantemente ao tratamento em seus estágios iniciais, tem resultado numa diminuição de até $90 \%$ nas taxas de incidência do câncer cervical invasor, quando o rastreamento apresenta boa cobertura e é realizado dentro dos padrões de qualidade (OMS, 2012); (Nascimento, et al., 2015).

A baixa adesão por parte das mulheres em maior risco para o desenvolvimento da doença, o despreparo das equipes de saúde para o enfretamento do problema, bem como a ausência de um sistema de informações que permita o adequado acompanhamento dessas mulheres estão entre os muitos desafios a serem enfrentados no rastreamento abrangente e efetivo do CCU (Slovinski, Slovinski \& Oliveira, 2020).

O Ministério da Saúde (2017), recomenda que o exame seja realizado principalmente em mulheres de 25 a 65 anos, pois se observa baixa incidência e mortalidade pelo CCU fora desta faixa etária. É de suma importância o diagnóstico na fase inicial da doença para que assim possa ser instituído o tratamento levando a paciente a cura (Silva, et al., 2019).

Nessa perspectiva, observa-se que um dos determinantes do diagnóstico de CCU em estágios avançados é a idade. Em seu estudo, Thuler e colaboradores (2014), apontam que para mulheres com idade entre 30 e 39 anos, a chance de desenvolvimento dessa patologia em sua forma mais avançada foi $10 \%$ maior do que em mulheres mais jovens, aumentando gradativamente até duas vezes para a faixa etária de 60 anos ou mais. De acordo Bruno, et al. (2019), há também um maior risco de aparecimento de carcinoma cervical invasivo em mulheres com idade superior a 60 anos. Isso acontece em decorrência da dificuldade de reconhecimento precoce de anomalias e alterações celulares que são frequentes no período pós menopausa.

Segundo Borges, et al. (2012), mulheres com idade acima de 59 anos possuem uma adesão diminuída a realização dos exames ginecológicos quando comparado a faixas etárias inferiores. Isso se dá devido a aproximação da menopausa, o que pode ocasionar um afastamento da mulher as práticas de prevenção ginecológica, em uma faixa etária em que os índices de CCU são cada vez maiores, que é compatível com os achados no presente estudo (Figura 1).

Moura e colaboradores (2010), em seu estudo, declaram que a procura pela realização do exame nos serviços de saúde diminui com a idade, quanto mais avançadas na idade, as mulheres realizam menos o exame, fazendo com que estas tornem-se um grupo de risco para esse tipo de câncer. Em relação a mulheres jovens e adolescentes, um dos principais problemas 
avaliados é que estas procuram realizar o exame de Papanicolau somente alguns anos depois de iniciada a atividade sexual, e após uma variada troca de parceiros.

A baixa adesão ao uso de preservativos bem como o medo ou vergonha das adolescentes e mulheres jovens para a realização do exame são grandes fatores de risco para essa faixa etária. A própria literatura cientifica aponta que as taxas de incidência de lesões cervicais e uterinas em adolescentes e mulheres jovens são alarmantes, e que embora sejam lesões majoritariamente de baixo grau podem invariavelmente na ausência de tratamento adequado progredir para lesões malignas, principalmente quando associadas a infecção pelo HPV (Dell'agnolo, et al., 2014); (Ribeiro \& Pereira, 2014).

Além da idade, outros fatores clínicos e epidemiológicos estão relacionados ao $\mathrm{CCU}$, destacando-se o início precoce da atividade sexual, fatores socioeconômicos como baixa escolaridade e renda, histórico de ISTs, tabagismo, uso prolongado de pílulas anticoncepcionais, carências nutricionais etc. (Almeida, Lobo \& Oliveira, 2018). A escolaridade é um fator importante nesse contexto. Andrade, et al., (2012) coloca a baixa escolaridade como fator que limita à adesão das mulheres ao exame. Logo, o nível de escolaridade certamente prejudica o entendimento das mulheres sobre a gravidade do CCU (Silva, et al., 2019).

Jorge e colaboradores (2011), relatam em sua pesquisa que o analfabetismo e o baixo nível educacional são condições que podem impedir ou dificultar o entendimento acerca do exame, sua necessidade de realização periodicamente, esclarecimento sobre a doença e a utilização dos serviços de saúde. Dessa forma, ações de promoção e prevenção de saúde ficam restritas ao entendimento e compreensão das mulheres. Nesse estudo, também é demonstrado que os indivíduos que possuem um grau mais elevado de ensino, detêm cuidados maiores com sua saúde e assim buscam o serviço com maior frequência. É importante ressaltar que as mulheres que apresentam uma baixa escolaridade possuem uma maior probabilidade de não realizar o exame, havendo como principal barreira a dificuldade de compreensão e entendimento sobre o CCU e o próprio teste de Papanicolau (Silva, et al., 2014).

O Ministério da Saúde (2017), aponta que inicialmente o exame de Papanicolau deve ser feito anualmente. Após dois exames seguidos (um por ano) com resultado normal, o preventivo pode passar a ser feito a cada três anos. Dessa forma, de acordo com a Figura 2, observa-se que muitas mulheres não seguem a estratégia de rastreamento proposta pelo MS e assim tornam-se mais susceptíveis ao desenvolvimento do CCU.

A proporção alta de mulheres que não realizam o exame anualmente também indica que ainda existe uma deficiência relacionada ao aspecto da prevenção, conforme recomendado pelo MS. Mulheres que nunca realizaram o exame ou que passam muito tempo sem realizá-lo estão mais propensas a desenvolver esse tipo de câncer (Azevedo, Cavalcante, Cavalcante \& Rolim, 2016). Além da prevenção, a repetição do exame após o primeiro teste tem como principal objetivo reduzir a possibilidade de resultados falso negativos na primeira rodada do rastreamento. A periodicidade de três anos deve-se a uma recomendação da OMS e às diretrizes adotadas por outros países (INCA, 2016).

O CCU pode ser diagnosticado na fase pré-clínica, a partir de medidas eficazes de rastreamento. O programa de rastreamento deve ser executado de forma organizada, seguindo ações previamente programadas, tendo como enfoque a população alvo de acordo com a faixa etária preconizada e a periodicidade de realização dos exames bem estabelecidas (Amaral, et al., 2014).

No Brasil, o rastreamento do CCU é tido como oportuníssimo, tendo em vista que as mulheres têm realizado o exame de Papanicolau quando procuram os serviços de saúde com outras motivações. Sendo assim, $20 \%$ a 25\% dos exames têm sido realizados fora do grupo etário recomendado e aproximadamente metade deles com intervalo de um ano ou menos, quando o recomendado são três anos. Desse modo, existe um contingente de mulheres super rastreadas e outra parte sem qualquer teste de rastreamento (INCA,2013). 
As diretrizes atuais para os casos com resultado de células escamosas atípicas de significado indeterminado (ASC-US) é a repetição da citologia em seis meses nas mulheres acima dos 30 anos, para mulheres entre 25 a 29 anos a repetição em um ano, e após três anos em mulheres com menos de 25 anos. O grupo ASC-US engloba alterações morfológicas insuficientes para determinar uma lesão intraepitelial. O seguimento de mulheres com ASC-US demonstra que elas apresentam risco aumentado para lesões em comparação às mulheres com resultado negativo para lesão intraepitelial e malignidade (INCA, 2016); (Rosendo, et al., 2018).

A importância do seguimento no CCU se dá pela detecção de complicações; detecção de lesões residuais; instituição de tratamento conservador; diminuição do risco de câncer pós-tratamento conservador e identificação de mulheres com menor necessidade de vigilância (INCA, 2010). Dessa forma, esse aspecto se constitui como um indicador de saúde e qualifica a assistência prestada à mulher (Farias \& Barbiere, 2016).

\section{Conclusão}

Diante do exposto conclui-se que a faixa etária mais prevalente de realização dos exames foi de 35 a 39 anos seguida pela idade de 40 a 44 anos. O rastreamento do câncer do colo do útero é uma ação complexa que se inicia com a definição da população alvo e finaliza com o diagnóstico das lesões suspeitas e tratamento das mulheres com câncer. O número de mulheres acometidas por essa patologia ainda é considerado alto e significativo. Nesse sentido, é fundamental identificar os fatores de risco e desenvolver estratégias que sejam eficientes no controle do CCU.

A realização de estudos como este, permite o reconhecimento dos fatores de risco para o CCU e fornece subsídios para melhor orientar, tratar e encaminhar ao serviço especializado, as mulheres com maior potencial de desenvolver o CCU, além de contribuir para a realização de mais pesquisas a respeito do tema em questão.

Diante da análise de dados foi possível constatar algumas dificuldades e limitações, como por exemplo os dados relacionados a escolaridade, considerado um fator de risco que influencia diretamente a não realização do exame preventivo por parte das mulheres. Sendo assim, é necessário discutir a qualidade das informações prestadas pelo Sistema de Informação do Câncer, tendo em vista que dados completos refletem melhor a realidade e influenciam na cobertura e rastreamento da população que realiza o exame preventivo.

Por fim, é notório que é necessário um melhor conhecimento sobre o tema em questão para o fortalecimento e redirecionamento das políticas de controle do câncer do colo uterino. Assim, o conhecimento epidemiológico possibilita fornecer um melhor acompanhamento dos grupos mais vulneráveis por meio de ações especificas.

\section{Referências}

Almeida, M. M., Lobo, L. M. D. G. A. \& Oliveira, F. B. M. (2018). Câncer do colo uterino, hpv e exame papanicolaou: uma reflexão acerca dos conhecimentos das mulheres. Revista Ciência \& Saberes-UniFacema. 4 (1), 889-95.

Amaral, A. F., Araújo, E. S., Magalhães, J. C., Silveira, É. A., Tavares, S. B. d. N., \& Amaral, R. G. (2014). Impacto da capacitação dos profissionais de saúde sobre o rastreamento do câncer do colo do útero em unidades básicas de saúde. Revista Brasileira de Ginecologia e Obstetrícia, 36(4), 182187. https://doi.org/10.1590/s0100-7203201400040004

Andrade, C. T. d., Magedanz, A. M. P. C. B., Escobosa, D. M., Tomaz, W. M., Santinho, C. S., Lopes, T. O., \& Lombardo, V. (2012). A importância de uma base de dados na gestão de serviços de saúde. Einstein (São Paulo), 10(3), 360-365. https://doi.org/10.1590/s1679-45082012000300018

Araújo, C. S, Luz, H. A \& Ribeiro, G. T. F. (2011). Exame preventivo de Papanicolau: percepção das acadêmicas de enfermagem de um centro universitário do interior de Goiás. Revista Mineira de Enfermagem, 15 (3), 378-85.

Azevedo, A. G., Cavalcante, I. B., Cavalcante, J. B. \& Rolim, L. A. D. M. M. (2016). Fatores que influenciam a não realização do exame de Papanicolaou e o impacto de ações educativas. Revista Brasileira de Análises Clínicas, 48, 253-57.

Backes, L. T. H., Bertolin, T. E., Manfredini, V., Klock, C., Calil, L. N., \& Mezzari, A. (2014). Alterações citológicas cervicovaginais no Alto Uruguai Gaúcho, Rio Grande do Sul. Revista de Ciências Médicas, 23(2), 65. https://doi.org/10.24220/2318-0897v23n2a2525 
Borges, M. F. d. S. O., Dotto, L. M. G., Koifman, R. J., Cunha, M. d. A., \& Muniz, P. T. (2012). Prevalência do exame preventivo de câncer do colo do útero em Rio Branco, Acre, Brasil, e fatores associados à não-realização do exame. Cadernos de Saúde Pública, 28(6), 1156-1166. https://doi.org/10.1590/s0102$311 \times 2012000600014$

Brasil (2016). Ministério da Saúde. Manual de gestão da qualidade para laboratório de citopatologia. Rio de Janeiro, RJ. http://www.saude.gov.br/images/pdf/2016/maio/12/livro-completo-manual-citopatologia.pdf.

Brasil (2017). Ministério da Saúde. Ministério da Saúde amplia faixa etária para rastreamento do câncer do colo do útero. Rio de Janeiro, RJ.

Brasil (2013). Ministério da Saúde. Secretaria de Atenção à Saúde. Departamento de Atenção Básica. Controle dos cânceres do colo do útero e da mama $/$ Ministério da Saúde, Secretaria de Atenção à Saúde, Departamento de Atenção Básica. - 2. ed. -Brasília: Editora do Ministério da Saúde.

Brasil (2017). Ministério da Saúde. Sistema de Informação do Câncer - SISCAN. Brasília, DF.

Bruno, M. T., Coco, A., Di Pasqua, S. \& Bonanno, G. (2019). Management of ASC-US/HPV positive post-menopausal woman. Virology journal, 16(1), 1-4 https://doi.org/10.1186/s12985-019-1145-5

De Carvalho, F. L. O., et al. (2018). HPV como principal precursor do câncer de colo de útero em adolescentes. Revista de Saúde ReAGES, 1 (2), 23 -36.

Dell'Agnolo, C. M., Brischiliari, S. C. R., Saldan, G., Gravena, A. A. F., Lopes, T. C. R., Demitto, M. d. O., \& Pelloso, S. M. (2014). Avaliação dos exames citológicos de papanicolau em usuárias do sistema único de saúde. Revista Baiana Saúde Pública, 38(4), 854-864. https://doi.org/10.5327/z0100-02332014380400007

Farias, A. C. B. D. \& Barbieri, A. R. (2016). Seguimento do câncer de colo de útero: estudo da continuidade da assistência à paciente em uma região de saúde. Escola Anna Nery, 20 (4).

Instituto Nacional De Câncer José Alencar Gomes Da Silva (INCA). (2020). Controle do Câncer do Colo do Útero: Conceito e Magnitude. Rio de Janeiro, RJ. https://www.inca.gov.br/controle-do-cancer-do-colo-do-utero/conceito-e-magnitude

Instituto Nacional De Câncer José Alencar Gomes Da Silva (INCA). (2016). Diretrizes Brasileiras para o Rastreamento do Câncer do Colo do Útero, 2. ed. rev. atual. Rio de Janeiro, https://www.inca.gov.br/sites/ufu.sti.inca.local/files//media/document//diretrizesparaorastreamentodocancerdocolodoutero_2016_corrigido.pdf

Instituto Nacional De Câncer José Alencar Gomes Da Silva (INCA). (2019). Estimativa 2020: Incidência de Câncer no Brasil. INCA, Rio de Janeiro, RJ. https://www.inca.gov.br/sites/ufu.sti.inca.local/files/media/document/estimativa-2020-incidencia-de-cancer-no-brasil.pdf

Instituto Nacional De Câncer José Alencar Gomes Da Silva (INCA). (2013). Monitoramento das ações de controle dos cânceres do colo do útero e de mama. Informativo Detecção Precoce, 4(1), jan./abr Rio de Janeiro, RJ. https://www.inca.gov.br/sites/ufu.sti.inca.local/files//media/document//informativo-deteccaoprecoce-1-2013.pdf

Instituto Nacional De Câncer José Alencar Gomes Da Silva (INCA). (2020). HPV e câncer: Perguntas mais frequentes. Rio de Janeiro, RJ. https://www.inca.gov.br/perguntas-frequentes/qual-e-relacao-entre-hpv-e-cancer

Instituto Nacional De Câncer José Alencar Gomes Da Silva (INCA). (2010). Plano de ação para redução da incidência e mortalidade por câncer do colo do útero. Rio de Janeiro, RJ. https://www.inca.gov.br/sites/ufu.sti.inca.local/files//media/document//plano-de-acao-para-reducao-da-incidencia-e-mortalidade-porcancer-do-colo-do-utero-2010.pdf

Instituto Nacional De Câncer José Alencar Gomes Da Silva (INCA). (2020). Tipos de Câncer: Câncer do Colo do Útero. Rio de Janeiro, RJ, 2020. https://www.inca.gov.br/tipos-de-cancer/cancer-do-colo-do-utero

Jorge, R. J. B., Diógenes, M. A. R., Mendonça, F. A. d. C., Sampaio, L. R. L., \& Jorge Júnior, R. (2011). Exame Papanicolaou: sentimentos relatados por profissionais de enfermagem ao se submeterem a esse exame. Ciência \& Saúde Coletiva, 16(5), 2443-2451. https://doi.org/10.1590/s141381232011000500013

Kose, F. M., \& Naki, M. M. (2014). Cervical premalignant lesions and their management. Journal of the Turkish German Gynecological Association, 15(2), 109-121. https://doi.org/10.5152/jtgga.2014.29795

Lopes, V. A. S., \& Ribeiro, J. M. (2019). Fatores limitadores e facilitadores para o controle do câncer de colo de útero: uma revisão de literatura. Ciência \& Saúde Coletiva, 24(9), 3431-3442. https://doi.org/10.1590/1413-81232018249.32592017

MINISTÉRIO DA SAÚDE. (2020). Departamento de Informática do SUS - DATASUS. Informações de Saúde, Epidemiológicas e Morbidade: banco de dados. http://tabnet.datasus.gov.br/cgi/dhdat.exe?SISCAN/cito_colo_residpi.def

Moura, A. D. A. (2010). Conhecimento e motivações das mulheres acerca do exame de papanicolau: subsídios para a para a prática de enfermagem. Rev. Rene, Fortaleza, 11(1), 94-104.

Nascimento, G. W. D. C., Pereira, C. C. D. A., Nascimento, D. I. D. C., Lourenço, G. C., \& Machado, C. J. (2015). Cobertura do exame citopatológico do colo do útero no Estado de Minas Gerais, Brasil, no período entre 2000-2010: um estudo a partir dos dados do Sistema de Informação do Câncer do Colo do Útero (SISCOLO). Cadernos Saúde Coletiva, 23(3), 253-260. https://doi.org/10.1590/1414-462x201500030059

Nóbrega, A. L., et al. (2014). A importância da assistência de enfermagem para a realização do exame citopatológico: um olhar bibliográfico. Informativo Técnico do Semiárido, 8(2), p. 01-08.

Pereira, A. S., Shitsuka, D. M., Parreira, F. J. \& Shitsuka, R. (2018). Metodologia da pesquisa científica. [e-book]. Santa Maria. Ed. UAB/NTE/UFSM.

Pinto, D. d. S., Fuzii, H. T., \& Quaresma, J. A. S. (2011). Prevalência de infecção genital pelo HPV em populações urbana e rural da Amazônia Oriental Brasileira. Cadernos de Saúde Pública, 27(4), 769-778. https://doi.org/10.1590/s0102-311x2011000400016 
Rangel, G., Lima, L. D. d., \& Vargas, E. P. (2015). Condicionantes do diagnóstico tardio do câncer cervical na ótica das mulheres atendidas no Inca. Saúde em Debate, 39(107), 1065-1078. https://doi.org/10.1590/0103-110420161070261

Rico, A. M., \& Iriart, J. A. B. (2013). "Tem mulher, tem preventivo": sentidos das práticas preventivas do câncer do colo do útero entre mulheres de Salvador, Bahia, Brasil. Cadernos de Saúde Pública, 29(9), 1763-1773. https://doi.org/10.1590/s0102-311x2013001300016

Rosendo, D. A., et al. (2018) Células escamosas atípicas de significado indeterminado (ASC-US): seguimento de exames analisados no Instituto Adolfo Lutz. RBAC, 50(3), 265-9.

Silva, D. S. M. d., Silva, A. M. N., Brito, L. M. O., Gomes, S. R. L., Nascimento, M. d. D. S. B., \& Chein, M. B. d. C. (2014). Rastreamento do câncer do colo do útero no Estado do Maranhão, Brasil. Ciência \& Saúde Coletiva, 19(4), 1163-1170. https://doi.org/10.1590/1413-81232014194.00372013

Silva, I. D., Da Silva, M. E. T., Andrade, J. S. d. O., Nunes, B. C. M., \& Pego, C. O. (2019). Exame papanicolau: percepção das mulheres sobre os motivos que influenciam a sua não realização. Revista Eletrônica Acervo Saúde, (34), Artigo e1125. https://doi.org/10.25248/reas.e1125.2019

Slovinski, B. G., Slovinski, J. G., \& Oliveira, H. R. (2020). Exame preventivo de colo do útero: análise do perfil das usuárias e dos dados de incidência de câncer. Fag journal of health (fjh), 2(2), 273-283. https://doi.org/10.35984/fjh.v2i2.160

Thuler, L. C. S., Aguiar, S. S. d., \& Bergmann, A. (2014). Determinantes do diagnóstico em estadio avançado do câncer do colo do útero no Brasil. Revista Brasileira de Ginecologia e Obstetrícia, 36(6), 237-243. https://doi.org/10.1590/s0100-720320140005010

Thuler, L. C. S., Bergmann, A., \& Casado, L. (2012). Perfil das Pacientes com Câncer do Colo do Útero no Brasil, 2000-2009: Estudo de Base Secundária. Revista Brasileira de Cancerologia, 58(3), 351-357. https://doi.org/10.32635/2176-9745.rbc.2012v58n3.583

WHO. World Health Organization. (2012). Câncer. Genebra [internet]. WHO: https://www.who.int/health-topics/cancer

WHO. World Health Organization. (2019). Folha informativa: HPV e câncer do colo do útero https://www.paho.org/bra/index.php?option=com_content\&view=article\&id=5634:folha-informativa-hpv-e-cancer-do-colo-do-utero\&Itemid=839 